\title{
REFERENCES
}

1. A. W. Goodman, The rotation theorem for starlike univalent functions, Proc. Amer. Math. Soc. vol. 4 (1953) pp. 278-286.

2. K. Löwner, Über Extremumsätze bei der konformen Abbildungen des Äusseren des Einheitskreises, Math. Zeit. vol. 3 (1919) pp. 65-77.

3. A. Marx, Untersuchungen über schlichte Abbildungen, Math. Ann. vol. 107 (1932) pp. 40-67.

4. R. M. Robinson, Univalent majorants, Trans. Amer. Math. Soc. vol. 61 (1947) pp. 1-35.

5. W. Stroganoff, Über den arc $f^{\prime}(z)$ unter der Bedingung, dass $f(z)$ die konforme Abbildung eines sternartigen Gebietes auf das innere des Einheitskreises der z-Ebene liefert, Trudy Mat. Inst. Steklov. vol. 5 (1934) pp. 247-258.

University of California, Berkeley

\section{CRITERIA OF BOUNDEDNESS OF THE SOLUTIONS OF NONLINEAR DIFFERENTIAL EQUATIONS}

\section{CHOY-TAK TAAM}

1. In this paper we shall use the following lemma to derive some criteria of boundedness of the solutions of certain nonlinear differential equations.

Lemma. Suppose that the following conditions are satisfied:

1. $u(x)$ and $v(x)$ are real-valued functions, defined and non-negative for $x \geqq a$,

2. $v(x)$ and $u(x) v(x)$ belong to $L(a, R)$ for every $R>a$,

3. for some positive constant $M$

$$
u(x) \leqq M+\int_{a}^{x} u(t) v(t) d t \quad(x \geqq a) .
$$

Then

$$
u(x) \leqq M \exp \left(\int_{a}^{x} v(t) d t\right) \quad(x \geqq a) .
$$

This lemma is useful in the study of boundedness and asymptotic behavior of the solutions of differential equations, see for instance $[1 ; 2 ; 3]$. Its proof is simple: divide the left-hand member of $(1.1)$ by its right-hand member and multiply the result by $v(x)$, then integrate

Presented to the Society, April 23, 1954; received by the editors April 19, 1954 and, in revised form, August 9, 1954. 
from $a$ to $x$, obtaining

$$
\log \left(M+\int_{a}^{x} u(t) v(t) d t\right) \leqq \log M+\int_{a}^{x} v(t) d t .
$$

The inequality (1.2) then follows from (1.1) and (1.3).

In the sequel we assume that the coefficients $r(x)$ and $p_{i}(x)$ of the differential equations discussed are real-valued continuous functions of the real variable $x$ on $a \leqq x<\infty, r(x)$ being positive. It is also assumed that each product $r(x) p_{i}(x)$ is absolutely continuous on $a \leqq x<\infty$. Only real-valued solutions which are not identically zero shall be considered.

2. In this section we shall prove two theorems concerning the nonlinear differential equation

$$
\left(r(x) y^{\prime}\right)^{\prime}+\sum_{i=1}^{n} p_{i}(x) y^{2 i-1}=0 .
$$

Denote by $f_{+}^{\prime}(x)$ the $\max \left(f^{\prime}(x), 0\right)$ and by $f_{-}^{\prime}(x)$ the $\min \left(f^{\prime}(x), 0\right)$.

THEOREM 1. Let the following conditions be satisfied:

1. $r p_{i}>0$ on $a \leqq x<\infty, i=1,2, \cdots, n$,

2. $\left(r p_{i}\right)^{-1}\left(r p_{i}\right)_{-}^{\prime}$ belongs to $L(a, \infty)$ for some $i=k$ and $\left(r p_{i}\right)^{-1}\left(r p_{i}\right)_{+}^{\prime}$ belongs to $L(a, \infty)$ for all $i \neq k$.

Then every solution of (2.1) is bounded on $a \leqq x<\infty$.

Proof. Write $f_{i}=r p_{i}$. Multiplying (2.1) by $2 r y^{\prime}$ we obtain

$$
2 r y^{\prime}\left(r y^{\prime}\right)^{\prime}+2 \sum_{i=1}^{n} f_{i} y^{2 i-1} y^{\prime}=0 .
$$

Integrating (2.2) from $a$ to $x$ and evaluating the result by integration by parts, we have

$$
\left(r y^{\prime}\right)^{2}+\sum_{i=1}^{n} i^{-1} f_{i} y^{2 i}=c+\sum_{i=1}^{n} i^{-1} \int_{a}^{x} f_{i}^{\prime} y^{2 i} d x,
$$

where $c$ is a positive constant. Clearly

$$
\sum_{i=1}^{n} i^{-1} f_{i} y^{2 i} \leqq c+\sum_{i=1}^{n} i^{-1} \int_{a}^{x}\left(f_{i}\right)_{+}^{\prime} y^{2 i} d x
$$

and so

$$
\sum_{i=1}^{n} i^{-1} f_{i} y^{2 i} \leqq c+\int_{a}^{x}\left[\sum_{i=1}^{n}\left(f_{i}\right)_{+}^{\prime} f_{i}^{-1}\right]\left[\sum_{i=1}^{n}{ }_{i}^{-1} f_{i} y^{2 i}\right] d x .
$$


It follows that from the lemma and (2.5) that

$$
\sum_{i=1}^{n} i^{-1} f_{i} y^{2 i} \leqq c \exp \left(\int_{a}^{x}\left[\sum_{i=1}^{n}\left(f_{i}\right)_{+}^{\prime} \bar{f}_{i}^{-1}\right] d x\right) \text {. }
$$

Since

$$
\int_{a}^{x}\left(f_{k}\right)_{+}^{\prime} f_{k}^{-1} d x=\log f_{k}-\log c_{1}-\int_{a}^{x}\left(f_{k}\right)_{-}^{\prime} f_{k}^{-1} d x,
$$

we have (from (2.6))

$$
f_{k} y^{2 k} \leqq c_{2} f_{k} \exp \left[-\int_{a}^{x}\left(f_{k}\right)_{-}^{\prime} f_{k}^{-1} d x+\sum_{i=1}^{n} \int_{a}^{x}\left(f_{i}\right)_{+}^{\prime} f_{i}^{-1} d x\right]
$$

for some positive constant $c_{2}$, where the summation sign $\sum^{\prime}$ does not include $i=k$. In view of the conditions in (2), the integrals on the right-hand side of (2.7) are bounded on $a \leqq x<\infty$. Hence $y$ is bounded on $a \leqq x<\infty$. This completes the proof.

We observe that conditions (1) and (2) imply that, for some $i=k$, $r p_{i}$ has a positive lower bound, and for all $i \neq k, r p_{i}$ has a positive upper bound.

In application, the following result may be more useful.

THEOREM 2. Let the following conditions be satisfied:

1. $r p_{i} \rightarrow a_{i}$ as $x \rightarrow \infty, a_{i}$ being positive constant, $i=1,2, \cdots, n$,

2. $\left(r p_{i}\right)^{\prime}$ belongs to $L(a, \infty), i=1,2, \cdots, n$.

Then, for every solution $y$ of (2.1), $y$ and ry' are bounded on $a \leqq x<\infty$.

Furthermore, in addition to conditions (1) and (2),

I. if a solution $y$ of (2.1) oscillates an infinite number of times on $a \leqq x<\infty$, then the maximum values of $|y|$ and those of $\left|r y^{\prime}\right|$ tend respectively to some limits $Y$ and $M$ as $x$ tends to infinity and

$$
M^{2}=\sum_{i=1}^{n} i^{-1} a_{i} Y^{2 i}
$$

II. if a solution $y$ of (2.1) oscillates only a finite number of times on $a \leqq x<\infty$, then $y$ and $r y^{\prime}$ both tend respectively to some limits as $x$ tends to infinity;

III. if there is a solution of (2.1) which tends to a limit different from zero as $x$ tends to infinity, then every solution $y$ of (2.1) and ry' both tend respectively to some limits as $x$ tends to infinity, and $r^{-1}$ and $p_{i}(i=1,2, \cdots, n)$ belong to $L(a, \infty)$;

IV. if $r^{-1}$ belongs to $L(a, \infty)$, then every solution $y$ of (2.1) and $r y^{\prime}$ both tend respectively to some limits as $x$ tends to infinity. 
Proof. Conditions (1) and (2) of this theorem imply conditions (1) and (2) of Theorem 1 for a sufficiently large $a$. The boundedness of $y$ then follows from Theorem 1. Also the boundedness of $r y^{\prime}$ follows from (2.3). Write (2.3) as

$$
\left(r y^{\prime}\right)^{2}+\sum_{i=1}^{n} i^{-1} a_{i} y^{2 i}=c_{1}+\epsilon(x),
$$

$c_{1}$ being a non-negative constant and

$$
\epsilon(x)=\sum_{i=1}^{n}\left[i^{-1}\left(a_{i}-f_{i}\right) y^{2 i}-i^{-1} \int_{x}^{\infty} f_{i}^{\prime} y^{2 i} d x\right] .
$$

Clearly $\epsilon(x)$ tends to zero as $x$ tends to infinity.

If a solution $y$ oscillates an infinite number of times on $a \leqq x<\infty$ and if $y_{j}$ is the maximum value of $|y|$ occurring at $x_{j}$, where $x_{1}, x_{2}, \cdots, x_{j}, \cdots$ are arranged in increasing order, then from (2.9) we have

$$
\lim _{y \rightarrow \infty} \sum_{i=1}^{n} i^{-1} a_{i} y_{j}^{2 i}=c_{1}
$$

which in turn implies that the sequence of maxima of $|y|$ tends to some limit $Y$. Similarly if $M_{1}, M_{2}, \cdots, M_{j}, \cdots$ are the maxima of $\left|r y^{\prime}\right|$ occurring at a set of values of $x$ arranged in increasing order, then $y=0$ for large $x$ in this set and from (2.9)

$$
\lim _{j \rightarrow \infty} M_{j}^{2}=c_{1}
$$

The equation (2.8) then follows from (2.11) and (2.12).

If a solution $y$ of (2.1) oscillates only a finite number of times on $a \leqq x<\infty$, then, for large $x, y$ remains one sign and it is easy to see, after integrating the equation (2.1) once, that $y$ is either increasing or decreasing. By virtue of its property of boundedness, $y$ tends to a limit as $x$ tends to infinity. From (2.9), $r y^{\prime}$ also tends to a limit as $x$ tends to infinity.

Integrating (2.1) from $a$ to $x$, we have

$$
\int_{a}^{x} \sum_{i=1}^{n} p_{i} y^{2 i-1} d x=r(a) y^{\prime}(a)-r(x) y^{\prime}(x) .
$$

In view of the boundedness of $r y^{\prime}$, the integral in (2.13) is also bounded on $a \leqq x<\infty$. If there is a solution $y_{0}$ of (2.1) which tends to a limit different from zero, then, for all $i$ and for large $x, p_{i} y_{0}^{2 i-1}$ 
and $\sum_{i=1}^{n} p_{i} y_{0}^{2 t-1}$ remain the same sign. It follows that for $y=y_{0}$ the integral in (2.13) is convergent as $x$ tends to infinity and hence

$$
\int_{a}^{\infty} p_{i} y_{0}^{2 i-1} d x \quad(i=1,2, \ldots, n)
$$

also exists. By virtue of the fact that $y_{0}$ has a limit different from zero as $x$ tends to infinity and $p_{i}$ is positive for large $x, p_{i}$ belongs to $L(a, \infty)$ for $i=1,2, \cdots, n$. Then $r^{-1}$ also belongs to $L(a, \infty)$, in view of the condition (1). Since $r y^{\prime}$ is bounded on $a \leqq x<\infty$ for an arbitrary solution $y, y^{\prime}$ belongs to $L(a, \infty)$ and hence $y$ tends to a limit as $x$ tends to infinity. That $r y^{\prime}$ also tends to a limit as $x$ tends to infinity is again a consequence of (2.9). This completes the proof.

Under conditions (1) and (2) of Theorem 2, the solutions of (2.1) may or may not oscillate an infinite number of times on $a \leqq x<\infty$. For example, no solution of

$$
\left(x^{2} y^{\prime}\right)^{\prime}+x^{-2} y+x^{-2} y^{3}=0
$$

oscillates an infinite number of times on $1 \leqq x<\infty$, while every solution of

$$
\left(x^{-2} y^{\prime}\right)^{\prime}+x^{2} y+x^{2} y^{3}=0
$$

does. Both equations can be reduced to

$$
d^{2} y / d t^{2}+y+y^{3}=0
$$

by change of independent variable, $t x=1$ for the first equation and $3 t=x^{3}$ for the second. The above remark then follows from the fact that every solution of (2.14) has only a finite number of zeros on the interval $0<t \leqq 1$ but infinite number of zeros on $1 \leqq t<\infty$.

3. In this section we consider the more general differential equation

$$
\left(r(x) y^{\prime}\right)^{\prime}+\sum_{i=1}^{n} p_{i}(x) y^{i}=0
$$

where $n$ is a positive odd integer, in the interval $\mathrm{I}: a \leqq x<\infty$.

THEOREM 3. Suppose that the following conditions are satisfied:

1. $\left(r p_{i}\right)^{\prime}$ belongs to $L(a, \infty), i=1,2, \cdots, n$,

2. $r p_{n}$ has a positive lower bound on $I$.

Then, for every solution $y$ of (3.1), $y$ and $r y^{\prime}$ are bounded on $I$.

Proof. The conditions of the theorem imply that $r p_{i}$, $i=1,2, \cdots, n$, is bounded on $I$ and tends to a limit $a_{i}$ as $x$ tends to 
infinity and $a_{n}>0$. Since $n+1$ is even, it follows that there exist constants $y_{0}$ and $A, y_{0}>1$ and $A>0$, such that for all $x$ on $I$

$$
\sum_{i=1}^{n}(i+1)^{-1} r p_{i} y^{i+1} \geqq A y^{n+1}
$$

whenever $|y| \geqq y_{0}$. We shall assume that $y_{0}$ is also greater than the least upper bound of the absolute values of the roots $z$ of the algebraic equation

$$
\sum_{i=1}^{n} p_{i}(x) z^{i}=0
$$

where $x$ varies in $I$. This is possible because the algebraic equation (3.3) is equivalent to

$$
\sum_{i=1}^{n} r p_{i} z^{i}=0
$$

and for all $x$ in $I$ the roots of $z$ of (3.4) are uniformly bounded, by virtue of the boundedness of $\left(r p_{i}\right)\left(r p_{n}\right)^{-1}, i=1,2, \cdots, n-1$, on $I$ [5].

Let $y(x)$ be an arbitrary solution of (3.1). If $|y(x)|$ is not already bounded by $y_{0}$ on $I$, let $|y(x)|-y_{0}$ change sign at the points

$$
x_{0}, x_{1}, x_{2}, \cdots, x_{i}, \cdots
$$

of $I$ which are arranged in increasing order, $|y(x)|-y_{0} \geqq 0$ on $\left[x_{2 k}, x_{2 k+1}\right]$ and $|y(x)|-y_{0} \leqq 0$ on $\left[x_{2 k+1}, x_{2 k+2}\right]$. We may suppose that the sequence (3.5) is not finite, otherwise either $|y(x)|$ is bounded by $y_{0}$ for large $x$ or it can be proved to be bounded on $I$ in a manner similar to the case of an infinite sequence. There are at most a finite number of points of (3.5) in any finite closed subinterval $I_{0}$ of $I$. To prove this, suppose that it is false. Then $I_{0}$ contains a limit point $x^{\prime}$ of (3.5) and $\left|y\left(x^{\prime}\right)\right|=y_{0}$. Either $y(x)=y_{0}$ or $y(x)=-y_{0}$ then holds for an infinite number of points of $I_{0}$ in the neighborhood of $x^{\prime}$. Suppose that the first case holds (the second case can be treated similarly). It follows that $y^{\prime}$ and hence $\left(r y^{\prime}\right)^{\prime}$ must have infinite number of zeros in the neighborhood of $x^{\prime}$, but then $\left(r y^{\prime}\right)^{\prime}=0$ at $x^{\prime}$ and from (3.1)

$$
\sum_{i=1}^{n} p_{i}\left(x^{\prime}\right)\left(y\left(x^{\prime}\right)\right)^{i}=0
$$

i.e., $y\left(x^{\prime}\right)=y_{0}$ is a root of the algebraic equation (3.3) for $x=x^{\prime}$. This contradicts the choice of $y_{0}$ and hence the sequence (3.5) has no finite limit point. 
In much the same way as in the proof of Theorem 1, we have (3.6) $\frac{1}{2}\left(r y^{\prime}\right)^{2}+\sum_{i=1}^{n} \frac{1}{i+1} r p_{i} y^{i+1}=c_{2 k}+\sum_{i=1}^{n} \frac{1}{i+1} \int_{x_{2 k}}^{x}\left(r p_{i}\right)^{\prime} y^{i+1} d x$, where $x$ lies on the interval $\left[x_{2 k}, x_{2 k+1}\right]$. On the interval $\left[x_{2 k}, x_{2 k+1}\right]$, where $|y(x)| \geqq y_{0}$, the inequality (3.2) is satisfied and $c_{2 k}$, being the value of the left-hand side of (3.6) at $x_{2 k}$, is positive. Set

$$
A q=\sum_{i=1}^{n} \frac{1}{i+1}\left|\left(r p_{i}\right)^{\prime}\right|
$$

Clearly

$$
\sum_{i=1}^{n} \frac{1}{i+1}\left|\left(r p_{i}\right)^{\prime} y^{i+1}\right| \leqq A q y^{n+1}
$$

for $x$ in $\left[x_{2 k}, x_{2 k+1}\right]$. It follows that from (3.2), (3.6), and (3.7)

$$
A y^{n+1} \leqq c_{2 k}+A \int_{x_{2 k}}^{x} q y^{n+1} d x
$$

holds for all $x$ in $\left[x_{2 k}, x_{2 k+1}\right]$. Using the lemma in $\$ 1$, we obtain

$$
A y^{n+1} \leqq c_{2 k} \exp \left(\int_{x_{2 k}}^{x} q d x\right)
$$

for $x$ in $\left[x_{2 k}, x_{2 k+1}\right]$. Let $c_{i}$ be the value of the left-hand member of (3.6) at the point $x_{i}$ of the sequence (3.5), $c_{i}$ being necessarily positive. Then from (3.6), (3.7), and (3.9), we have

$$
\begin{aligned}
c_{2 k+1} & \leqq c_{2 k}+A \int_{x_{2 k}}^{x_{2 k+1}} q y^{n+1} d x \\
& \leqq c_{2 k}+c_{2 k} \int_{x_{2 k}}^{x_{2 k+1}} q \exp \left(\int_{x_{2 k}}^{x} q d x\right) d x \\
& =c_{2 k} \exp \left(\int_{x_{2 k}}^{x_{2 k+1}} q d x\right) .
\end{aligned}
$$

On the interval $\left[x_{2 k+1}, x_{2 k+2}\right]$, where $|y(x)| \leqq y_{0}$, we have

$$
\begin{aligned}
& \frac{1}{2}\left(r y^{\prime}\right)^{2}+\sum_{i=1}^{n} \frac{1}{i+1} r p_{i} y^{i+1} \\
&=c_{2 k+1}+\sum_{i=1}^{n} \frac{1}{i+1} \int_{x_{2 k+1}}^{x}\left(r p_{i}\right)^{\prime} y^{i+1} d x .
\end{aligned}
$$


Setting

$$
v=\sum_{i=1}^{n} \frac{1}{i+1}\left|\left(r p_{i}\right)^{\prime}\right| y_{0}^{i+1}
$$

we have

$$
\begin{aligned}
c_{2 k+2} & =c_{2 k+1}+\sum_{i=1}^{n} \frac{1}{i+1} \int_{x_{2 k+1}}^{x_{2 k+2}}\left(r p_{i}\right)^{\prime} y^{i+1} d x \\
& \leqq c_{2 k} \exp \left(\int_{x_{2 k}}^{x_{2 k+1}} q d x\right)+\int_{x_{2 k+1}}^{x_{2 k+2}} v d x .
\end{aligned}
$$

Using (3.12) and mathematical induction, it is now easy to verify that

$$
c_{2 k} \exp \left(\int_{x_{2 k}}^{x_{2 k+1}} q d x\right) \leqq\left(c_{0}+\int_{x_{0}}^{x_{2 k+1}} v d x\right) \exp \left(\int_{x_{0}}^{x_{2 k+1}} q d x\right)
$$

for all $k$. Since the right-hand side of (3.13) is bounded for all $k$, it follows that, from (3.9), $y(x)$ is bounded on $I$. In (3.6) let $x$ be any point of $I$; the boundedness of $r y^{\prime}$ then follows from the boundedness of other terms in (3.6). This completes the proof of Theorem 3.

4. Most standard classical existence theorems assure us the existence and uniqueness of the solutions of the equations (2.1) and (3.1) only in the neighborhood of the points in question. In the proofs of the theorems in $\$ 2$ and $\S 3$ we assume implicitly the existence of the solutions on the interval $a \leqq x<\infty$ and part of our argument relies on the assumption of the uniqueness of the solutions on $a \leqq x<\infty$. To justify our assumptions, we point out that if, in addition to the conditions stated in Theorem 1 or Theorem 3, the assumption of the existence and uniqueness of a solution on $a \leqq x<\infty$ implies its boundedness on $a \leqq x<\infty$, then we can extend the solution to the whole interval $a \leqq x<\infty$ from its existence and uniqueness in a bounded interval and the extension is unique. In [4], a more detailed discussion of this point is given.

The literature about boundedness of solutions and related problems is extensive. Further references can be found in [1] which surveys the results in this area up to 1948.

The inequality (2.7) in the proof of Theorem 1 is an extension of a result of Bellman and Caccioppoli $[1$, p. 91]. As a corollary of Theorem 1, we observe that condition (2) of Theorem 1 is satisfied if $\left(r p_{k}\right)^{\prime} \geqq 0$ and $\left(r p_{i}\right)^{\prime} \leqq 0$ for all $i \neq k$. This corollary generalizes a known result (see [1, p. 92]) different from that obtained by Butlewski (see $[1$, p. 95]). However a result more general than this corollary 
and that of Butlewski has been obtained in [4] which proves that every solution of $(2.1)$ is bounded on $a \leqq x<\infty$ if each product $r p_{i}$ is monotonic and non-negative on $a \leqq x<\infty$ and if either (a) for at least one $i, r p_{i}$ is nondecreasing and positive on $a \leqq x<\infty$ or (b) for all $i, r p_{i}$ is nonincreasing and, for some $i, r p_{i}$ has a positive lower bound on $a \leqq x<\infty$, even $1 / r$ and $p_{i}$ are only Lebesgue integrable over $[a, x]$ for every $x>a$. Theorem 2 (I) is a result similar to that of Ascoli for linear equations $[1$, p. 128]. The author has not been able to find results similar to Theorem 3 in the literature.

\section{REFERENCES}

1. R. Bellman, $A$ survey of the theory of the boundedness, stability, and asymptotic behavior of solutions of linear and nonlinear differential and difference equations, Washington, D. C., Department of the Navy, 1949.

2. C. T. Taam, Linear differential equations with small perturbations, Duke Math. J. vol. 20 (1953) pp. 13-26.

3. - Asymptotic relations between systems of differential equations, submitted to the Pacific Journal of Mathematics.

4. - An extension of Osgood's oscillation theorem for a nonlinear differential equation, Proc. Amer. Math. Soc. vol. 5 (1954) pp. 705-715.

5. G. D. Birkhoff, An elementary double inequality for the roots of an algebraic equation having greatest absolute value, Bull. Amer. Math. Soc. vol. 21 (1915) pp. 494495.

The Catholic University of America 